\title{
A duality based proof of the Combinatorial Nullstellensatz
}

\author{
Omran Kouba \\ Department of Mathematics \\ Higher Institute for Applied Sciences and Technology \\ P.O. Box 31983, Damascus, Syria \\ omran_kouba@hiast.edu.sy
}

Submitted: Dec 21, 2008; Accepted: Mar 5, 2009; Published: Mar 13, 2009

Mathematics Subject Classifications: 05A99, 15A03

\begin{abstract}
In this note we present a proof of the combinatorial nullstellensatz using simple arguments from linear algebra.
\end{abstract}

The combinatorial nullstellensatz [1] is an elegant tool which has many applications in combinatorial number theory, graph theory and combinatorics (see [1] and [2]). In this note we present a proof of this result using simple arguments from linear algebra. In Theorem 1, we recall the statement of the combinatorial nullstellensatz:

Theorem 1. Let $P$ be a polynomial in $m$ variables $X_{1}, X_{2}, \ldots, X_{m}$ over an arbitrary field $\mathbb{K}$. Suppose that the coefficient of the monomial $X_{1}^{n_{1}} X_{2}^{n_{2}} \cdots X_{m}^{n_{m}}$ in $P$ is nonzero, and that the total degree of $P$ is $\sum_{j=1}^{m} n_{j}$. Then, if $S_{1}, S_{2}, \ldots, S_{m}$ are subsets of $\mathbb{K}$ such that card $\left(S_{j}\right)>n_{j}($ for $1 \leq j \leq n$,$) there is some \left(t_{1}, t_{2}, \ldots, t_{m}\right)$ in $S_{1} \times S_{2} \times \cdots \times S_{m}$ so that $P\left(t_{1}, t_{2}, \ldots, t_{m}\right) \neq 0$.

Our proof is based upon a simple lemma concerning linear forms on the vector space $\mathbb{K}[T]$ of polynomials in one variable $T$ over an arbitrary field $\mathbb{K}$. In the dual space $(\mathbb{K}[T])^{*}$, we consider the dual basis $\left(\varphi_{m}\right)_{m \geq 0}$ of the canonical basis $\left(T^{m}\right)_{m \geq 0}$ of $\mathbb{K}[T]$, this means that $\varphi_{m}(P)$ is the coefficient of $\bar{T}^{m}$ in $P$, in other words $\varphi_{i}\left(T^{j}\right)=\delta_{i j}$ where $\delta_{i j}$ is the Kronecker symbol. We also denote by $\mathbb{K}_{n}[T]$ the subspace of $\mathbb{K}[T]$ formed of polynomials of degree at most $n$.

With the above notation we have the following lemma :

Lemma 2. Let $S$ be a subset of $\mathbb{K}$ such that card $(S)=m+1$. Then there is a family $\left(\lambda_{t}^{S}\right)_{t \in S}$ of elements in $\mathbb{K}$ such that

$$
\forall P \in \mathbb{K}_{m}[T], \quad \varphi_{m}(P)=\sum_{t \in S} \lambda_{t}^{S} P(t)
$$


Proof. Consider, for $t \in S$, the linear form $\mu_{t}: \mathbb{K}_{m}[T] \longrightarrow \mathbb{K}, \mu_{t}(P)=P(t)$. The family $\left(\mu_{t}\right)_{t \in S}$ constitutes a basis of the dual space $\left(\mathbb{K}_{m}[T]\right)^{*}$. (To see this, note that if $\left(\ell_{t}\right)_{t \in S}$ denotes the basis of $\mathbb{K}_{m}[T]$ formed by the Lagrange intepolation polynomials : $\ell_{t}(T)=\prod_{s \in S \backslash\{t\}} \frac{T-s}{t-s}$, then $\mu_{u}\left(\ell_{v}\right)=\delta_{u v}$. This proves that $\left(\mu_{t}\right)_{t \in S}$ is the dual basis of $\left.\left(\ell_{t}\right)_{t \in S} \cdot\right)$

Now, the linear form $P \mapsto \varphi_{m}(P)$ defines an element from $\left(\mathbb{K}_{m}[T]\right)^{*}$ and, consequently, it has a unique expression as a linear combination of the elements of the basis $\left(\mu_{t}\right)_{t \in S}$. This proves the existence of a familly of scalars $\left(\lambda_{t}^{S}\right)_{t \in S}$, such that $\varphi_{m}(P)=\sum_{t \in S} \lambda_{t}^{S} \mu_{t}(P)$ for any polynomial $P$ in $\mathbb{K}_{m}[T]$, and achieves the proof of Lemma 2.

Before proceeding with the proof of Theorem 1, let us recall that the total degree of a polynomial $P$ from $\mathbb{K}\left[X_{1}, \ldots, X_{m}\right]$ is the largest value of $d_{1}+d_{2}+\cdots+d_{m}$ taken over all monomials $X_{1}^{d_{1}} X_{2}^{d_{2}} \cdots X_{m}^{d_{m}}$ with nonzero coefficients in $P$.

Proof of Theorem 1. For each $j$ in $\{1, \ldots, m\}$, we may assume that card $\left(S_{j}\right)=n_{j}+1$ (by discarding the extra elements if necessary,) then, using Lemma 2, we find a familly of scalars $\left(\lambda_{t}^{S_{j}}\right)_{t \in S_{j}}$ such that

$$
\forall P \in \mathbb{K}_{n_{j}}[T], \quad \varphi_{n_{j}}(P)=\sum_{t \in S_{j}} \lambda_{t}^{S_{j}} P(t)
$$

Then, we consider the linear form $\Phi$ on $\mathbb{K}\left[X_{1}, \ldots, X_{m}\right]$ defined by :

$$
\Phi(Q)=\sum_{\left(t_{1}, \ldots, t_{m}\right) \in S_{1} \times \cdots \times S_{m}} \lambda_{t_{1}}^{S_{1}} \lambda_{t_{2}}^{S_{2}} \cdots \lambda_{t_{m}}^{S_{m}} Q\left(t_{1}, t_{2}, \ldots, t_{m}\right) .
$$

Clearly, we have

$$
\begin{aligned}
\Phi\left(X_{1}^{d_{1}} X_{2}^{d_{2}} \cdots X_{m}^{d_{m}}\right) & =\sum_{t_{1} \in S_{1}} \sum_{t_{2} \in S_{2}} \cdots \sum_{t_{m} \in S_{m}} \lambda_{t_{1}}^{S_{1}} \lambda_{t_{2}}^{S_{2}} \cdots \lambda_{t_{m}}^{S_{m}} t_{1}^{d_{1}} t_{2}^{d_{2}} \ldots t_{m}^{d_{m}} \\
& =\prod_{j=1}^{m}\left(\sum_{t \in S_{j}} \lambda_{t}^{S_{j}} t^{d_{j}}\right) .
\end{aligned}
$$

So we have the following two properties:

$i$. If there is some $k$ in $\{1, \ldots, m\}$ such that $d_{k}<n_{k}$, then by (1) we have

$$
\sum_{t \in S_{k}} \lambda_{t}^{S_{k}} t^{d_{k}}=\varphi_{n_{k}}\left(T^{d_{k}}\right)=0
$$

and therefore, $\Phi\left(X_{1}^{d_{1}} X_{2}^{d_{2}} \cdots X_{m}^{d_{m}}\right)=0$. 
ii. On the other hand,

$$
\Phi\left(X_{1}^{n_{1}} X_{2}^{n_{2}} \cdots X_{m}^{n_{m}}\right)=\prod_{j=1}^{m} \varphi_{n_{j}}\left(T^{n_{j}}\right)=1 .
$$

Let us suppose that

$$
P=\sum_{\left(d_{1}, d_{2}, \ldots, d_{m}\right) \in \mathcal{D}} b_{d_{1}, d_{2}, \ldots, d_{m}} X_{1}^{d_{1}} X_{2}^{d_{2}} \cdots X_{m}^{d_{m}}
$$

where we collected in $\mathcal{D}$ the multi-indexes $\left(d_{1}, d_{2}, \ldots, d_{m}\right)$ satisfying $b_{d_{1}, d_{2}, \ldots, d_{m}} \neq 0$.

Now, if $\left(d_{1}, d_{2}, \ldots, d_{m}\right)$ is an element from $\mathcal{D}$ which is different from $\left(n_{1}, n_{2}, \ldots, n_{m}\right)$, then there is some $k$ in $\{1, \ldots, m\}$ such that $d_{k}<n_{k}$ because $\operatorname{deg}(P)=\sum_{j=1}^{m} n_{j}$. Therefore, by $\left(i\right.$.), if $\left(d_{1}, d_{2}, \ldots, d_{m}\right)$ is an element from $\mathcal{D}$ which is different from $\left(n_{1}, n_{2}, \ldots, n_{m}\right)$, then $\Phi\left(X_{1}^{d_{1}} X_{2}^{d_{2}} \cdots X_{m}^{d_{m}}\right)=0$, and if we use (ii.) we conclude that

$$
\Phi(P)=\sum_{\left(d_{1}, d_{2}, \ldots, d_{m}\right) \in \mathcal{D}} b_{d_{1}, d_{2}, \ldots, d_{m}} \Phi\left(X_{1}^{d_{1}} X_{2}^{d_{2}} \cdots X_{m}^{d_{m}}\right)=b_{n_{1}, n_{2}, \ldots, n_{m}} \neq 0 .
$$

Finally, the conclusion of the theorem follows since

$$
\sum_{\left(t_{1}, \ldots, t_{m}\right) \in S_{1} \times \cdots \times S_{m}} \lambda_{t_{1}}^{S_{1}} \lambda_{t_{2}}^{S_{2}} \cdots \lambda_{t_{m}}^{S_{m}} P\left(t_{1}, t_{2}, \ldots, t_{m}\right)=\Phi(P) \neq 0 .
$$

This ends the proof of Theorem 1.

\section{REFERENCES}

[1] Alon, N., Combinatorial Nullstellensatz. Recent trends in combinatorics. (Mátraháza, 1995). Combin. Probab. Comput. 8 (1999), 7-29.

[2] Shirazi, H. and Versträ̈te, J., A note on polynomials and $f$-factors of graphs. Electronic J. of Combinatorics, 15 (2008), \#N22. 